\title{
Differential survival after traumatic spinal cord injury: evidence from a multi-center longitudinal cohort study in Switzerland
}

\author{
Jonviea D. Chamberlain ${ }^{1,2} \cdot$ Hans Peter Gmünder ${ }^{3} \cdot$ Kerstin Hug $^{4} \cdot$ Xavier Jordan ${ }^{5} \cdot$ André Moser $^{6,7} \cdot$ \\ Martin Schubert ${ }^{8} \cdot$ Martin W.G. Brinkhof $\mathbb{D}^{1,2}$
}

Received: 20 February 2018 / Revised: 17 May 2018 / Accepted: 17 May 2018 / Published online: 12 June 2018

(C) International Spinal Cord Society 2018

\begin{abstract}
Study design Observational cohort study.

Objectives To understand differentials in the force of mortality with increasing time since injury according to key spinal cord injury (SCI) characteristics.

Setting Specialized rehabilitation centers within Switzerland.

Methods Data from the Swiss Spinal Cord Injury (SwiSCI) cohort study were used to model mortality in relation to age, sex, and lesion characteristics. Hazard ratios (HRs) and adjusted survival curves were estimated using flexible parametric survival models of time since discharge from first rehabilitation to death or 30 September 2011, whichever came first.

Results 2421 persons were included that incurred a new TSCI between 1990 and 2011, contributing a total time-at-risk of 19,604 person-years and 376 deaths. Controlling for attained age, sex, decade, and etiology, there was more than a four-fold higher risk of mortality for complete tetraplegia compared to incomplete paraplegia (HR $=4.27 ; 95 \%$ CI 2.72 to 6.69). Survival estimates differed according to SCI characteristics, with differentials steadily increasing with time since injury.

Conclusion This study provides evidence of disparities in mortality and survival outcomes according to SCI characteristics that increases with increasing time since injury. These results lend support to the hypothesis of a progressive and disproportionate accumulation of allostatic load according to SCI characteristics. Future research should investigate causespecific mortality for insight into potentially modifiable secondary health conditions contributing to these disparities.
\end{abstract}

Electronic supplementary material The online version of this article (https://doi.org/10.1038/s41393-018-0163-2) contains supplementary material, which is available to authorized users.

Jonviea D. Chamberlain

jonviea.chamberlain@paraplegie.ch

Swiss Paraplegic Research, Nottwil, Switzerland

2 University of Lucerne, Health Sciences and Health Policy, Luzern, Switzerland

3 Swiss Paraplegic Center, Nottwil, Switzerland

4 REHAB Basel, Basel, Switzerland

5 Clinique Romande de Réadaptation, Sion, Switzerland

6 Department of Geriatrics Bern, University Hospital, Spital Netz Bern Ziegler, University of Bern, Bern, Switzerland

7 Institute of Social and Preventative Medicine, University of Bern, Bern, Switzerland

8 Balgrist University Hospital, Zürich, Switzerland

\section{Introduction}

Traumatic spinal cord injuries (TSCIs) are a life-altering condition associated with serious monetary, social, and health-related burden that can accumulate over time resulting in reduced health outcomes and life expectancy. Within-population comparisons of all-cause mortality, a measure of disease burden, can support the identification of high-risk groups requiring targeted interventions to improve survival outcomes. Identifying risk factors contributing to associated burdens of TSCI on individual health is crucial for activating resources towards prevention and improvement of health outcomes, increased well-being, reduced socioeconomic inequalities, and in particular reduction in avoidable, or premature, mortality [1].

Extant literature has identified key SCI-related factors associated with an increased risk of mortality following a TSCI, particularly high (e.g., C1-C4) and complete lesions [2-4]. Recent evidence has found variation in risk of death with the prevalence of self-reported secondary health 
conditions (HCs) according to lesion characteristics, as well as an overall higher occurrence of reported HCs with increasing injury severity [5]. The accumulation of allostatic load associated with HCs according to lesion characteristics could contribute to the observed discrepancies in mortality estimates [4]. Understanding the within-population evolution of discrepancies in mortality and survival estimates could provide evidence for the accumulation of allostatic load and subsequent differentials in survival estimates with increasing time since injury.

Comparisons of mortality estimates across settings can serve to highlight competencies or deficiencies of health systems that could potentially inform other health systems towards improving long-term outcomes. Although recent large-scale studies in developed countries indicate broadly consistent survival outcomes, previous research has demonstrated discrepancies in the magnitude of risk for mortality following TSCI across settings, highlighting the influence of health systems on mortality and survival outcomes [4]. Therefore, country-specific and comparable estimates of mortality and longevity after SCI are needed. Additionally, within-SCI population comparisons of mortality and survival estimates can serve to identify potential modifiable factors associated with increased risk of mortality. The purpose of this study is to thus provide the first, Swiss-specific estimates of mortality and survival outcomes after TSCI using information collected in the SwiSCI cohort study. Moreover, this study aims to investigate the associated force of mortality within the SCI population with increasing time since injury.

\section{Methods}

\section{Study description}

The SwiSCI study is a longitudinal cohort study that aims to understand how to support "functioning, health maintenance, and quality-of-life of persons with SCI", and has been extensively described previously [6-8]. The present study uses data collected from the Medical Record study, encompassed within the broader SwiSCI study, which include data collected from medical records from before 1970 up until as recently as 2013 [6, 7]. The present study includes persons who incurred a new SCI between 1990 until 2011, given a recent vital status update for these individuals. Study eligibility criteria include persons admitted to first rehabilitation in one of the five SCI specialized rehabilitation centers (operational: REHAB Basel; Balgrist University Hospital; Swiss Paraplegic Centre; Clinique Romande de Réadaption; historic: University Hospital Geneva), with permanent residency in Switzerland at admission to specialized rehabilitation, aged
16 years or older at time of injury, and who did not incur SCI due to a congenital condition (e.g., spina bifida) or neurodegenerative disorder (e.g., multiple sclerosis). Data collected for the SwiSCI study underwent ethical review, and were approved by the ethical committees of Cantons: Lucerne, Basel, Valais, and Zürich (reference numbers: 1008 [Luzern]; 37/11 [Basel]; CCVEM 015/11 [Valais]; 2012-0049 [Zürich]). When investigating AIS grade and injury severity (categorical variable combining lesion level and AIS grade) as risk factors for premature mortality, only cases with an incurred TSCI after 2000 were included due to data unavailability and incompleteness of data collection pre-2000. Individuals with an AIS grade E were included within the analysis given their eligibility for inclusion in the overall SwiSCI study. AIS grades E and $\mathrm{D}$ were combined due to relatively few cases of AIS grade $\mathrm{E}(N=23)$.

\section{Data management}

All variables were grouped according to ISCOS guidelines to facilitate comparability with other studies [9]. Information on lesion type, level, completeness, and AIS score were collected at discharge from first rehabilitation. If data at discharge were missing, when available, data collected at admission to first rehabilitation were used instead $(N=10)$.

\section{Outcome evaluation}

The outcome of interest for this study was death as of 30 September 2011. In 2011, the first Community Survey (CS 2012) for SwiSCI participants started recruitment based on potential participants identified through the Medical Records study [8]; 30 September 2011 is chosen as the arbitrary start of the CS 2012, when questionnaires were initially sent out. Although the present study only uses information from the Medical Records study, active participation, or response to the CS 2012 questionnaire facilitated an update for the vital status of many individuals within the Medical Records study. Missing vital status information for individuals who did not respond to the CS 2012, or who were not included or eligible, was updated in a subsequent tracing effort. This tracing effort updated vital status first through specialized clinics, and if needed through community of last known residence (similar to the study methodology reported for the Swiss Childhood Cancer cohort [10]). Individuals were identified as either alive, dead, or lost to follow-up (LTFU) (i.e., no information on vital status) as of 30 September 2011. Given that cauda equina lesions are peripheral lesions with a different prognosis and evolution, cases of cauda equina were excluded from all survival analysis. 


\section{Statistical analysis}

The study population was described by frequencies $(n)$, percentages (\%), person-years (PYRS), mean and standard deviation (SD). Differences between injury cohorts were assessed by a chi-squared test or Kruskal-Wallis test. Kaplan-Meier curves were estimated in order to have a nonparametric assessment of survival probabilities, thereby making no assumptions regarding underlying survival distribution. Stratification by secondary variable was used for adjustment of Kaplan-Meier curves for known confounders that could impact survival (e.g., age, lesion level, and lesion completeness). Log-rank tests were used to test the equality of Kaplan-Meier curves. Kaplan-Meier survival estimates according to years since injury are presented in the supplementary material (Supplementary Table 2). We then used flexible parametric proportional hazards models to investigate risk factors for dying to estimate HRs and survival probabilities with 95\% confidence intervals (CI) [11]. Timeat-risk started at date of injury, with study entry defined as date of admission to first rehabilitation, and study exit as date of death or 30 September 2011, whichever came first. We reported unadjusted HRs, as well as HRs adjusted for sex, age, decade of TSCI, and cause of TSCI. In a secondary analysis, data were split on follow-up time to account and estimate the effect of follow-up time on mortality and longevity outcomes. These follow-up periods were:

1. After admission until discharge from first rehabilitation

2. One year post discharge

3. More than one year post discharge

Flexible parametric models are intended to model the baseline hazard in a way that allows flexibility in the shape of the survival distribution modeled; this flexibility comes from the use of restricted cubic splines [11]. The flexibility of the spline function is dictated by the number of knots, or points at which the baseline hazard is allowed to change. The minimal AIC and BIC values were used to formally select the number of knots included in the model. Violations to the proportional hazards function, implicit to flexible parametric models (as well as other survival models), were assessed using likelihood ratio test for time-dependent effects of included covariates. Survival curves were estimated using direct adjustment; with direct adjustment, survival curves - for categorical variables - are estimated at specified time points for a combination of covariate patterns, to provide a final average of these values at each time point. This is importantly different to the mean covariate method, which provides survival estimates according to the variable of interest, but while using the mean value of all underlying covariates-an issue for categorical variables [11]. The absolute difference between estimated survival probabilities adjusted for potential confounders was estimated so as to demonstrate the impact of lesion characteristics (i.e., completeness and lesion level) on survival probabilities. The figures provided show the difference in survival between selected characteristics of interest (e.g., complete tetraplegic lesions and incomplete paraplegic lesions) if they had the same covariate distribution as that of the whole study population (i.e., the same underlying distributions in age, sex, lesion level, and cause of TSCI).

To account for age effects that occur due to biological processes of aging, age was time-updated using splitting techniques so that, as each individual aged, the individual contributed different amounts of time to each risk set. For example, if an individual was injured at the age of 29 and subsequently died at the age of 33 , this individual would contribute one year of follow-up time to the age category "16-30 years" and roughly three years to the age group, "31-45 years". Similarly, to account for potential period effects that could result from changes in medical technology and rehabilitation approaches, data were further split on decade of injury; again allowing for an individual to contribute to different risk sets (e.g., 1990-1999 or 2000-2011).

In a sensitivity analysis to account for persons LTFU, inverse probability weights (IPW) were estimated using logistic regression including LTFU (yes/no) as the outcome and decade of SCI, sex, age at injury, lesion level, rehabilitation center, and completeness of lesion as independent variables in the model. For those missing complete information on independent variables, the mean weight for the total population was used.

We reported $p$-values from two-sided test statistics. A $p$ value smaller than 0.05 was considered as significant. All analyses were implemented in STATA software version 14.2 [12].

\section{Results}

The study population is described in Table 1 . Between 1990 and 2011, 2421 persons incurred a traumatic spinal cord injury and were eligible for inclusion in the present study. Of these individuals, $73.2 \%$ were male, $42.2 \%$ were paraplegic (excluding cauda equina), and the average age at injury was 44.6 years $(\mathrm{SD}=19.4$; $\mathrm{IQR}=32)$. Between the first (1990-1999) and second decade (2000-2011), the average age at injury increased by roughly five years (41.5 years and 46.6 years, respectively). Significant differences were observed between decades according to age at injury, length of stay, etiology of injury, type of SCI, and completeness. For example, in the latter decade there was a larger proportion of TSCIs with a complete lesion (70.9\% compared to $63.0 \%$ ), fewer transport-related TSCIs $(25.9 \%$ 
Table 1 Demographic and SCI-specific characteristics of participants by injury cohort

\begin{tabular}{|c|c|c|c|}
\hline \multirow[t]{2}{*}{ Characteristics } & \multicolumn{3}{|l|}{ Injury cohorts } \\
\hline & $\begin{array}{l}1990-1999 \\
(n=950)\end{array}$ & $\begin{array}{l}2000-2011 \\
(n=1471)\end{array}$ & $P$-value \\
\hline $\begin{array}{l}\text { Age at injury, years: } \\
\text { mean; S.D. (IQR) }\end{array}$ & $41.5 ; 18.5(29)$ & $46.6 ; 19.6(33)$ & $<0.001$ \\
\hline $\begin{array}{l}\text { Length of stay, } \\
\text { months: mean; S.D. } \\
\text { (IQR) }\end{array}$ & $6.2 ; 9.9(4.7)$ & $5.2 ; 4.4(4.3)$ & $<0.001$ \\
\hline $\operatorname{Sex}(1)$ & & & 0.24 \\
\hline Male & $708(74.5)$ & $1064(72.4)$ & \\
\hline Female & $242(25.5)$ & $406(27.6)$ & \\
\hline Age at injury $(0)$ & & & $<0.001$ \\
\hline $16-30$ & $351(36.9)$ & $385(26.2)$ & \\
\hline $31-45$ & $227(23.9)$ & $372(25.3)$ & \\
\hline $46-60$ & $191(20.1)$ & $310(21.1)$ & \\
\hline $61-75$ & $125(13.2)$ & $261(17.7)$ & \\
\hline $76+$ & $56(5.9)$ & $143(9.7)$ & \\
\hline Etiology (2) & & & $<0.001$ \\
\hline Sports and leisure & $186(19.6)$ & $359(24.4)$ & \\
\hline Transport & $326(34.4)$ & $381(25.9)$ & \\
\hline Falls & $324(34.2)$ & $555(37.7)$ & \\
\hline Other cause & $112(11.8)$ & $176(12.0)$ & \\
\hline SCI Type (12) & & & $<0.01$ \\
\hline Tetra & $531(56.2)$ & $776(53.0)$ & \\
\hline Para & $343(36.3)$ & $611(41.7)$ & \\
\hline Cauda equine & $71(7.5)$ & $77(5.3)$ & \\
\hline Completeness (95) & & & $<0.001$ \\
\hline Complete & $566(63.0)$ & $1012(70.9)$ & \\
\hline Incomplete & $332(37.0)$ & $416(29.1)$ & \\
\hline $\begin{array}{l}\text { Lesion level \& } \\
\text { completeness (52) }\end{array}$ & & & $<0.001$ \\
\hline $\begin{array}{l}\text { Paraplegia, } \\
\text { incomplete }\end{array}$ & $283(33.5)$ & $479(35.1)$ & \\
\hline Paraplegia, complete & $235(27.8)$ & $288(21.1)$ & \\
\hline $\begin{array}{l}\text { Tetraplegia, } \\
\text { incomplete }\end{array}$ & $229(27.1)$ & $473(34.7)$ & \\
\hline Tetraplegia, complete & $97(11.5)$ & $125(9.2)$ & \\
\hline ASIA score (943) & & & 0.75 \\
\hline AIS A & $60(28.6)$ & $380(29.8)$ & \\
\hline AIS B & $24(11.4)$ & $160(12.5)$ & \\
\hline AIS C & $32(15.2)$ & $214(16.8)$ & \\
\hline AIS D/E & $94(44.8)$ & $521(40.9)$ & \\
\hline $\begin{array}{l}\text { Injury severity } \\
*(1 \text { 292) }\end{array}$ & & & N.A. \\
\hline $\mathrm{C} 1-\mathrm{C} 4 \mathrm{ABC}$ & - & $108(10.3)$ & \\
\hline C5-C8 ABC & - & $97(9.3)$ & \\
\hline T1-S3 ABC & $3(3.1)$ & $318(30.5)$ & \\
\hline AIS D/E & $94(96.9)$ & $521(49.9)$ & \\
\hline
\end{tabular}

Table 1 (continued)

\begin{tabular}{|c|c|c|c|}
\hline \multirow[t]{2}{*}{ Characteristics } & \multicolumn{3}{|c|}{ Injury cohorts } \\
\hline & $\begin{array}{l}1990-1999 \\
(n=950)\end{array}$ & $\begin{array}{l}2000-2011 \\
(n=1471)\end{array}$ & $P$-value \\
\hline $\begin{array}{l}\text { Destination after } \\
\text { discharge (69) }\end{array}$ & & & 0.22 \\
\hline Private residence & $709(80.0)$ & $1146(78.2)$ & \\
\hline Hospital & $58(6.5)$ & $80(5.5)$ & \\
\hline $\begin{array}{l}\text { Nursing home/ } \\
\text { assisted living }\end{array}$ & $89(10.0)$ & $184(12.6)$ & \\
\hline Other (e.g., hotel) & $4(0.5)$ & $13(0.9)$ & \\
\hline Death & $26(2.9)$ & 43 (2.9) & \\
\hline $\begin{array}{l}\text { Ventilator } \\
\text { assistance (73) }\end{array}$ & & & 0.26 \\
\hline No & $881(96.8)$ & $1379(95.9)$ & \\
\hline Yes & $29(3.2)$ & $59(4.1)$ & \\
\hline $\begin{array}{l}\text { Associated injuries } \\
\left(\begin{array}{l}1 \\
1\end{array} 51\right)\end{array}$ & & & N.A. \\
\hline Yes & - & $321(56.3)$ & \\
\hline No & - & 249 (43.7) & \\
\hline
\end{tabular}

SCI Spinal cord injury, AIS American Spinal Injury Association (ASIA) Impairment Scale (AIS)

* $p$-value from chi-squared test or Kruskal-Wallis test

compared to $34.4 \%$ ), and a larger proportion of older individuals over the age of 60 years old admitted to specialized rehabilitation (27.4\% compared to $19.1 \%$ ). There was no difference between decades were observed for sex, American Spinal Injury Association (ASIA) Impairment Scale (AIS) score, destination after discharge, and ventilator assistance (Table 1). The majority of injuries were due to falls $(36.3 \%)$ and transport-related incidents $(29.2 \%)$. The total contributing time-at-risk over the study period was 19,604 PYRS (median 7.1 years), with 376 recorded deaths of which 67 occurred during the rehabilitation period (see Supplementary Table).

\section{Risk factors for mortality}

Attained age, TSCI type, completeness of lesion, and cause of TSCI were associated with an increased risk of mortality (Table 2A). Sex or decade of injury were not associated with risk of mortality. In the analyses stratified according to follow-up time, risk factors for mortality remained relatively stable in terms of the direction of effect, but varied slightly in magnitude. For example, while falls or 'other source of injury' (e.g., surgical)—compared to a transport-related TSCI-were identified as a significant risk factor for early mortality, estimated HRs were attenuated or non-significant during the inpatient period and first year post-discharge (Table 2B). The overall variation in risk was mainly driven by 
Table 2A Univariable and multivariable hazard ratios from the flexible parametric survival model

\begin{tabular}{llll}
\hline Characteristic & $\begin{array}{l}\text { Univariable } \\
\text { analysis } \\
\text { hazard ratio } \\
(95 \% \text { CIs })\end{array}$ & $\begin{array}{l}\text { Multivariable } \\
\text { analysis } \\
\text { hazard ratio } \\
(95 \% \text { CIs })\end{array}$ \\
\hline
\end{tabular}

\section{Model one}

Sex

Female

Male

Reference

$1.38(1.10-1.74)$

Reference

Attained age

$16-30$

$31-45$

$46-60$

$61-75$

$76+$

Reference

2.60 (1.36-4.98)

$5.46(2.93-10.19)$

14.59

(8.03-26.52)

43.15

(23.83-78.13)

Lesion Level

Paraplegia

Tetraplegia

Completeness

Incomplete

Complete

Decade of SCI

1990-1999

2000-2011

Cause of TSCI

Transport-related

Sports/Leisure

activity

Fall

Other

Model two

Lesion Level \&

Completeness

Paraplegia,

incomplete

Paraplegia,

complete

Tetraplegia,

incomplete

Tetraplegia,

complete

Model three

Injury severity ${ }^{\mathrm{a}}$

AIS D/E

C1-C4 ABC

C5-C8 ABC

T1-S3 ABC

0.99

$<0.001$

Reference

2.53 (1.32-4.85)

$5.20(2.77-9.77)$

13.21

(7.14-24.43)

38.27

(20.53-71.33)

$<0.001$

Reference Reference

$2.03(1.64-2.51) \quad 1.74(1.38-2.20)$

Reference Reference

$1.00(0.80-1.25)$

$2.08(1.64-2.65)$

Reference Reference

$1.52(1.14-2.02) \quad 1.10(0.82-1.47)$

Reference Reference

$0.88(0.59-1.31)$

$0.93(0.62-1.39)$

$3.20(2.41-4.25)$

$2.51(1.73-3.63)$

$1.51(1.12-2.04)$

$1.66(1.13-2.45)$

$<0.001$

Reference Reference

$1.11(0.80-1.53) \quad 1.54(1.11-2.14)$

$2.04(1.55-2.68) \quad 1.50(1.00-2.27)$

$2.34(1.67-3.29) \quad 4.27(2.72-6.69)$

$<0.01$

$\begin{array}{ll}\text { Reference } & \text { Reference } \\ 3.31(1.83-5.98) & 2.43(1.29-4.58) \\ 2.09(1.07-4.10) & 3.27(1.62-6.62) \\ 1.59(0.96-2.64) & 2.04(1.18-3.52)\end{array}$

aAnalyses including "Injury Severity" are restricted to 2000-2011 due to inadequate data prior to 2000. Model one is adjusted for: current age, sex, decade of TSCI, cause of TSCI, lesion level, and completeness of injury; Model two is adjusted for: current age, sex, decade of TSCI, cause of TSCI, and "Lesion level and completeness"; and Model three is adjusted for: current age, sex, injury severity, and cause of TSCI the one year or more post-discharge mortality. A similar pattern of attenuated effect sizes in comparison with the inpatient period was observed for attained age, particularly during the period of less than one year post discharge (e.g., for individuals aged 76 years and older: Overall $\mathrm{HR}=38.3,95 \%$ CI 20.5 to 71.3 [Table 2A]; one year post discharge $\mathrm{HR}=$ $27.7,95 \%$ CI 7.3 to 104.4 [Table 2B]). Contrarily, the risk for mortality increased for complete tetraplegia during the period of one year post discharge, and then subsequently decreased during the period greater than one year post discharge. However, due to the limited number of deaths during the inpatient period $(N=67)$ and the period of one year post discharge $(N=68)$, the power to capture the true relationship is limited, as demonstrated in the wide confidence intervals. There was evidence for interaction between TSCI type and completeness of lesion $(p \leq 0.01)$, although not between attained age and sex, or attained age and cause of TSCI. Given the evidence for an interaction, in a secondary analysis based on a categorical variable of a combination of completeness and level of injury, individuals with complete tetraplegic lesions had the highest risk of mortality when compared to subjects with incomplete paraplegic lesions $(\mathrm{HR}=4.27,95 \% \mathrm{CI}=2.72-6.69)$ (Table 2A).

\section{Survival probabilities}

Differences in Kaplan-Meier survival curves were observed according to sex $(p<0.001)$, age $(p<0.001)$, type of TSCI $(p<0.001)$, and injury severity (a combination of lesion level and AIS score) $(p<0.001)$. When controlling for attained age, a difference was additionally observed for completeness $(p<$ $0.001)$, although no longer according to sex $(p=0.60)$. Kaplan-Meier survival curves for completeness and level of lesion are presented in Fig. 1; stratified estimates according to years since follow-up are reported in Supplementary Table 2. In comparison with paraplegia, adjusted survival probabilities, estimated using flexible parametric survival models, for tetraplegia were diminished, with the divergence increasing over time (Table 3). For example, a $4.2 \%$ difference between survival probabilities for paraplegia $(93.4 \%$; $95 \%$ CI 91.6 to 95.2\%) compared to tetraplegia $(89.2 \%$; $95 \%$ CI 86.7 to $91.8 \%$ ) was observed at one year post admission, while for 20 -year survival estimates the difference grew to nearly $10 \%$ (Table 3). The increasing trend results in an overall augmentation in the discrepancy between survival probabilities for paraplegia compared to tetraplegia of $5.6 \%$ overall (Fig. 2). The discrepancy was even more pronounced for estimates according to level and completeness of lesion, whereas there was a $11.6 \%$ increase in the gap between complete tetraplegia and incomplete paraplegia at one-year survival (12.7\% difference) and 20-year survival (24.3\% difference) (Table 3). The absolute difference in survival probabilities for complete tetraplegia compared to incomplete 
Table 2B Risk factors according to follow-up period: Multivariable results from flexible parametric survival model

\begin{tabular}{|c|c|c|c|c|c|c|}
\hline Characteristic & $\begin{array}{l}\text { Inpatient } \\
\text { rehabilitation }\end{array}$ & $P$-value & $\begin{array}{l}<1 \text { year post- } \\
\text { discharge }\end{array}$ & $P$-value & $\begin{array}{l}\geq 1 \text { year post- } \\
\text { discharge }\end{array}$ & $P$-value \\
\hline \multicolumn{7}{|l|}{ Model one } \\
\hline Sex & & 0.66 & & 0.71 & & 0.74 \\
\hline Female & Reference & & Reference & & Reference & \\
\hline Male & $1.13(0.65-1.96)$ & & $1.11(0.64-1.95)$ & & $0.95(0.70-1.28)$ & \\
\hline Attained age & & $<0.001$ & & $<0.001$ & & $<0.001$ \\
\hline $16-30$ & Reference & & Reference & & Reference & \\
\hline $31-45$ & $1.99(0.44-8.94)$ & & $\begin{array}{l}2.48 \\
(0.61-10.07)\end{array}$ & & $2.85(1.18-6.90)$ & \\
\hline $46-60$ & $6.60(1.77-24.53)$ & & $\begin{array}{l}6.02 \\
(1.60-22.63)\end{array}$ & & $\begin{array}{l}5.14 \\
(2.15-12.30)\end{array}$ & \\
\hline $61-75$ & $\begin{array}{l}14.81 \\
(4.16-52.72)\end{array}$ & & $\begin{array}{l}12.41 \\
(3.41-45.19)\end{array}$ & & $\begin{array}{l}13.75 \\
(5.85-32.32)\end{array}$ & \\
\hline $76+$ & $\begin{array}{l}63.75 \\
(17.78-228.52)\end{array}$ & & $\begin{array}{l}27.68 \\
(7.34-104.41)\end{array}$ & & $\begin{array}{l}39.03 \\
(16.46-92.59)\end{array}$ & \\
\hline TSCI type & & 0.03 & & $<0.01$ & & $<0.001$ \\
\hline Paraplegia & Reference & & Reference & & Reference & \\
\hline Tetraplegia & $1.92(1.05-3.50)$ & & $2.49(1.38-4.49)$ & & 1.57 (1.18-2.09) & \\
\hline Completeness & & $<0.001$ & & $<0.01$ & & $<0.001$ \\
\hline Incomplete & Reference & & Reference & & Reference & \\
\hline Complete & $2.71(1.57-4.67)$ & & $2.53(1.40-4.59)$ & & $1.97(1.46-2.67)$ & \\
\hline Decade of SCI & & 0.09 & & 0.75 & & 0.07 \\
\hline 1990-1999 & Reference & & Reference & & Reference & \\
\hline 2000-2011 & $0.64(0.39-1.08)$ & & $1.10(0.60-2.03)$ & & $1.53(0.95-2.45)$ & \\
\hline Cause of TSCI & & 0.99 & & 0.15 & & $<0.01$ \\
\hline $\begin{array}{l}\text { Transport- } \\
\text { related }\end{array}$ & Reference & & Reference & & Reference & \\
\hline $\begin{array}{l}\text { Sports/Leisure } \\
\text { activity }\end{array}$ & $1.13(0.46-2.80)$ & & $0.57(0.20-1.64)$ & & $1.01(0.61-1.67)$ & \\
\hline Fall & $1.06(0.54-2.06)$ & & $1.09(0.54-2.21)$ & & $1.81(1.24-2.64)$ & \\
\hline Other & $1.01(0.34-3.01)$ & & $1.94(0.82-4.61)$ & & $1.76(1.08-2.84)$ & \\
\hline \multicolumn{7}{|l|}{ Model two } \\
\hline TSCI type & & $<0.001$ & & $<0.001$ & & $<0.001$ \\
\hline $\begin{array}{l}\text { Paraplegia, } \\
\text { incomplete }\end{array}$ & Reference & & Reference & & Reference & \\
\hline $\begin{array}{l}\text { Paraplegia, } \\
\text { complete }\end{array}$ & $1.74(0.69-4.40)$ & & $0.82(0.30-2.26)$ & & $1.71(1.17-2.51)$ & \\
\hline $\begin{array}{l}\text { Tetraplegia, } \\
\text { incomplete }\end{array}$ & $1.36(0.60-3.09)$ & & $1.33(0.68-2.59)$ & & $1.42(1.00-2.01)$ & \\
\hline $\begin{array}{l}\text { Tetraplegia, } \\
\text { complete }\end{array}$ & $4.52(1.91-10.74)$ & & $\begin{array}{l}6.91 \\
(3.22-14.83)\end{array}$ & & $3.36(2.10-5.37)$ & \\
\hline \multicolumn{7}{|l|}{ Model three } \\
\hline Injury severity ${ }^{a}$ & & 0.34 & & $<0.001$ & & 0.35 \\
\hline AIS D/E & Reference & & Reference & & Reference & \\
\hline $\mathrm{C} 1-\mathrm{C} 4 \mathrm{ABC}$ & $3.00(0.79-11.48)$ & & $\begin{array}{l}7.10 \\
(1.92-26.30)\end{array}$ & & $1.47(0.53-4.06)$ & \\
\hline C5-C8 ABC & $2.04(0.37-11.23)$ & & $\begin{array}{l}15.86 \\
(4.17-60.35)\end{array}$ & & $2.49(0.84-7.41)$ & \\
\hline $\mathrm{T} 1-\mathrm{S} 3 \mathrm{ABC}$ & $2.61(0.67-10.16)$ & & $\begin{array}{l}6.20 \\
(1.91-20.16)\end{array}$ & & $1.25(0.59-2.65)$ & \\
\hline
\end{tabular}

aModel three including "Injury Severity" only includes cases of TSCI post-2000. The model used for estimating hazard ratios in Model three including follow-up time less than one year post discharge specifies 2 knots, rather than the 3 knots used in all other models, as well as attained age as a continuous variable to aid in model convergence 


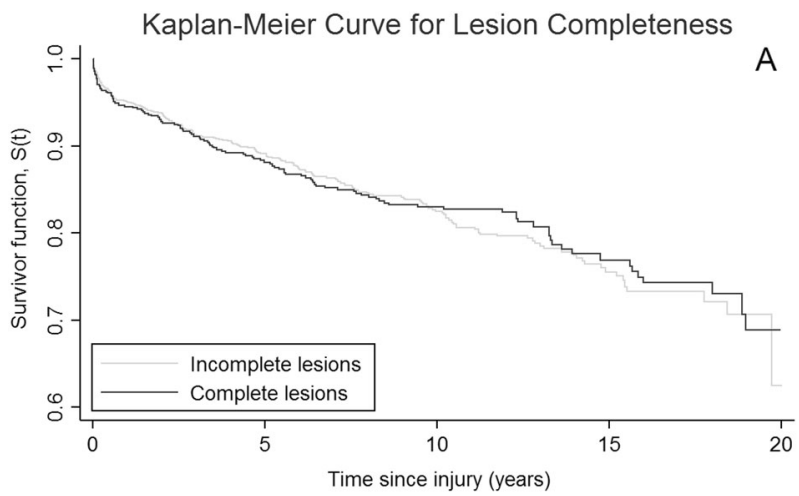

Kaplan-Meier Curve for Lesion Level

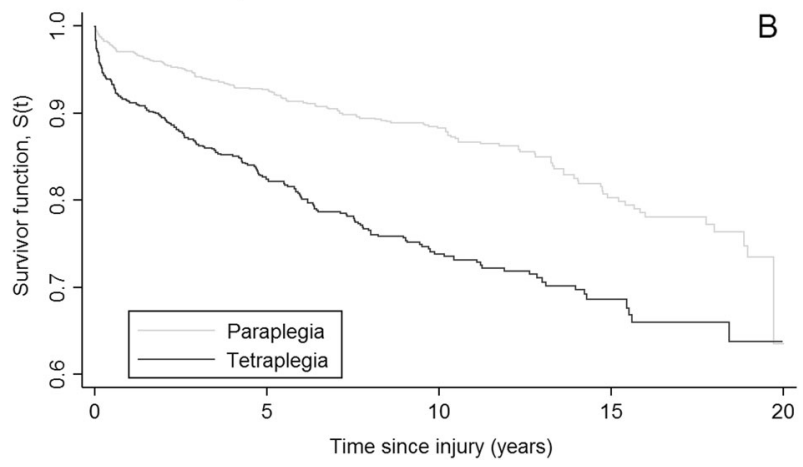

Fig. 1 Kaplan-Meier curves of the survival function stratified by SCI characteristics. a represents the Kaplan-Meier estimates for lesion completeness. b represents the Kaplan-Meier estimates for lesion level

paraplegia, standardized for underlying population characteristics, are presented in Fig. 2. Accounting for attained age, as a proxy for biological aging, the survival of complete tetraplegia was $4.8 \%$ (95\% CI 1.8-7.9\%) below that of incomplete paraplegia in absolute, standardized terms at 10 years (Fig. 2c). This implies that at 10 years, roughly $5 \%$ of the difference in survival probabilities is attributable to the difference in level and completeness of lesion. This discrepancy was slightly larger when adjusting for age at injury, rather than attained age (Supplementary figures).

\section{Discussion}

Older age, tetraplegia, completeness of lesion, and TSCIs due to falls or 'other causes' are associated with an increased risk of mortality following TSCI in Switzerland. Disparities in mortality and survival estimates according to SCI-specific characteristics were found to augment with increasing time since injury. Additionally, stratified results based on follow-up time post-injury suggest the potential modification in the magnitude of mortality risk according to sociodemographic and SCI-specific characteristics.

\section{Disparities in survival outcomes}

As individuals age with spinal cord injuries, there is an increasing gap in survival outcomes according to SCIspecific characteristics. It should be noted, however, that due to the limited follow-up time (i.e., 21 years) it is not known whether this trend towards a widening gap would continue, and whether it would continue at a similar rate. However, although comparison with previous literature is limited due to few studies providing stratified estimates, or only presenting evidence in figures of unadjusted Kaplan-Meier curves, of those studies that have provided stratified estimates for long-term follow-up have found similar results [2, 3, 13]. For example, a 50-year longitudinal study by Middleton et al. estimated a five-year survival for individuals with paraplegia and tetraplegia of $98 \%$ and $94 \%$, respectively; after fifteen years, the twentyyear survival dropped to $88 \%$ and $78 \%$. This represents an augmentation in the gap between survival estimates of $6 \%$ ( $4 \%$ between 5 -year estimates to $10 \%$ between 20 -year estimates); the present study estimated an increase of $3.7 \%$, including those who survived the first year, whereas Middleton et al excluded those that perished during the first year [2]. Discrepancies in survival estimates according to SCI-specific characteristics with increasing follow-up time could be due to the disproportionate accumulation of secondary HCs. It should also be noted that due to the limited follow-up time (i.e., 21 years), it is not known whether this trend towards a widening gap would continue, and whether it would continue at a similar rate; additional long-term studies investigating this question are needed to confirm whether or not this pattern continues. Furthermore, in order to investigate the influence of secondary HCs accumulation, or allostatic load, on mortality differentials according to SCI-specific characteristics, follow-up studies including time-updated information on secondary HCs are required.

Biological plausibility studies have suggested an increased immunological strain on the SCI-affected individual, and premature onset of immune frailty [14, 15], with animal experiments suggesting a dependency on lesion level [16]. Evidencing this, epidemiological studies have shown that individuals aging with SCI report a higher frequency of HCs [17]. Additionally, in the recent SwiSCI 2012 Community Survey, lesion type and completeness have been shown to play a role as individuals with higher, more severe lesions reported HCs more often, and reported different HCs [5]. For example, community members with a complete SCI reported a higher frequency of pressure ulcers, and members with tetraplegia reported more respiratory problems [5]. Secondary $\mathrm{HCs}$ - such as pressure injuries, depression, and infections-have been shown to be associated with an increased risk of mortality [18, 19], 
Table 3 Marginally adjusted survival probabilities according to time since injury

\begin{tabular}{|c|c|c|c|c|c|}
\hline & 1 -year & 5-year & 10-year & 15-year & 20-year \\
\hline \multicolumn{6}{|l|}{ Model one } \\
\hline Overall & 91.5 (89.6-93.5) & $86.2(84.4-88.1)$ & $81.2(79.4-83.1)$ & $77.1(75.0-79.2)$ & $73.7(71.2-76.3)$ \\
\hline \multicolumn{6}{|l|}{ Sex } \\
\hline Male & $91.5(89.5-93.5)$ & $86.2(84.3-88.2)$ & $81.2(79.2-83.2)$ & $77.1(74.8-79.4)$ & $73.7(71.0-76.5)$ \\
\hline Female & $91.5(89.2-93.9)$ & $86.3(83.8-88.9)$ & $81.3(78.4-84.3)$ & $77.2(73.9-80.6)$ & $73.8(70.0-77.8)$ \\
\hline \multicolumn{6}{|l|}{ Attained age } \\
\hline $16-30$ & $98.8(98.1-99.5)$ & 97.9 (96.7-99.1) & $96.9(95.1-98.7)$ & $95.9(93.6-98.2)$ & $95.0(92.1-97.9)$ \\
\hline $31-45$ & 97.1 (95.9-98.2) & 94.8 (93.1-96.6) & $92.3(90.0-94.7)$ & $90.0(87.0-93.1)$ & $87.8(84.2-91.6)$ \\
\hline $46-60$ & $94.1(92.0-96.1)$ & 89.7 (86.9-92.5) & $84.9(81.3-88.7)$ & $80.6(76.2-85.2)$ & $76.7(71.5-82.3)$ \\
\hline $61-75$ & 85.7 (81.6-90.0) & $76.1(71.3-81.2)$ & $66.6(61.2-72.5)$ & $58.7(52.8-65.4)$ & $52.3(45.7-59.7)$ \\
\hline $76+$ & $64.3(56.2-73.6)$ & $46.6(39.0-55.6)$ & $32.9(26.2-41.3)$ & $23.9(17.9-31.8)$ & $17.9(12.6-25.5)$ \\
\hline \multicolumn{6}{|l|}{ SCI type } \\
\hline Tetraplegia & $89.2(86.7-91.8)$ & $82.8(80.3-85.3)$ & $76.8(74.1-79.6)$ & $72.0(68.9-75.3)$ & $68.1(64.5-72.0)$ \\
\hline Paraplegia & $93.4(91.6-95.2)$ & $89.0(87.0-91.0)$ & 84.7 (82.5-86.9) & $81.0(78.5-83.6)$ & $77.9(75.0-80.9)$ \\
\hline \multicolumn{6}{|l|}{ Completeness } \\
\hline Incomplete & 92.9 (91.2-94.6) & $88.3(86.6-90.1)$ & $84.0(82.1-85.9)$ & $80.3(78.1-82.6)$ & $77.3(74.7-80.0)$ \\
\hline Complete & 86.8 (83.7-90.0) & 79.7 (76.7-82.9) & $73.5(70.3-76.8)$ & $68.6(65.2-72.2)$ & $64.8(61.0-68.8)$ \\
\hline \multicolumn{6}{|l|}{ Model two } \\
\hline \multicolumn{6}{|c|}{ Lesion level \& completeness } \\
\hline Paraplegia, incomplete & $93.3(91.4-95.3)$ & $89.0(86.7-91.3)$ & $84.8(82.2-87.6)$ & $81.4(78.4-84.5)$ & $78.6(75.3-82.0)$ \\
\hline Paraplegia, complete & $90.3(87.5-93.2)$ & 84.5 (81.3-87.9) & $79.3(75.7-83.1)$ & $75.1(71.2-79.2)$ & $71.7(67.6-76.2)$ \\
\hline Tetraplegia, incomplete & 91.9 (89.9-94.0) & $86.9(84.7-89.1)$ & $82.2(79.7-84.8)$ & $78.4(75.5-81.4)$ & $75.3(71.9-78.7)$ \\
\hline Tetraplegia, complete & $80.6(76.0-85.6)$ & $71.6(66.7-76.8)$ & $64.2(59.1-69.8)$ & $58.7(53.2-64.7)$ & $54.3(48.6-60.8)$ \\
\hline \multicolumn{6}{|l|}{ Model three } \\
\hline \multicolumn{6}{|l|}{ Injury severity ${ }^{a}$} \\
\hline $\mathrm{C} 1-\mathrm{C} 4 \mathrm{ABC}$ & $93.1(89.3-97.1)$ & $85.0(78.9-91.6)$ & 78.7 (70.8-87.6) & $74.4(65.0-85.2)$ & $71.0(60.4-83.5)$ \\
\hline $\mathrm{C} 5-\mathrm{C} 8 \mathrm{ABC}$ & $91.0(85.9-96.4)$ & $81.2(73.4-89.8)$ & $74.0(64.4-85.0)$ & $69.2(58.2-82.1)$ & $65.5(53.5-80.2)$ \\
\hline T1-S3 ABC & $94.1(91.3-97.1)$ & $87.0(82.7-91.5)$ & $81.3(75.5-87.4)$ & $77.2(70.1-85.0)$ & $74.0(65.8-83.3)$ \\
\hline AIS D/E & $96.8(95.2-98.5)$ & $92.6(90.0-95.2)$ & $88.8(85.2-92.4)$ & $85.9(81.2-90.8)$ & $83.5(77.8-89.6)$ \\
\hline
\end{tabular}

aAnalyses including "Injury Severity" are restricted to 2000-2011 due to inadequate data prior to 2000. Model one is adjusted for: Current age, sex, decade of TSCI, cause of TSCI, lesion level, and completeness of injury; Model two is adjusted for: Current age, sex, decade of TSCI, cause of TSCI, and "Lesion level and completeness"; Model three is adjusted for: Current age, sex, injury severity, and cause of TSCI

likely due to sepsis [20]. However, as evidenced in the recent nomenclature change from pressure ulcer to pressure injury, pressure injuries are preventable [21]. Identifying secondary HCs associated with cause-specific mortality can highlight potential areas to target interventions of modifiable risk factors for mortality, towards reducing the disparity in survival outcomes within the SCI population. Prospective studies are thus needed that simultaneously measure $\mathrm{HCs}$ and mortality.

\section{Comparisons within SCI literature}

Differences in the results of the current study in comparison with previous studies, as well as differences between previous studies could be influenced by contextual factors, for example, access to care, particularly when considering lower-income countries [4]. In order to exploit these discrepancies to improve mortality and survival outcomes and identify modifiable contextual factors associated with nonmodifiable risk factors of mortality (e.g., TSCI lesion level, completeness, severity, age, or sex), comparative estimates are essential. Presently, there are a number of aspects hampering comparability of estimates in SCI literature; namely, denotation of time-at-risk, definitions for acute mortality, differences in reference groups, and statistical approaches to analysis. For example, a study by Middleton et al. starts risk with date of SCI, uses a period of one year post injury for acute mortality, and estimates long-term survival after excluding those who did not survive one year post injury [2]. The aforementioned study by Cao et al. similarly excludes those who perished within the first year post-injury [22]. Another study by Hagen et al. defines 


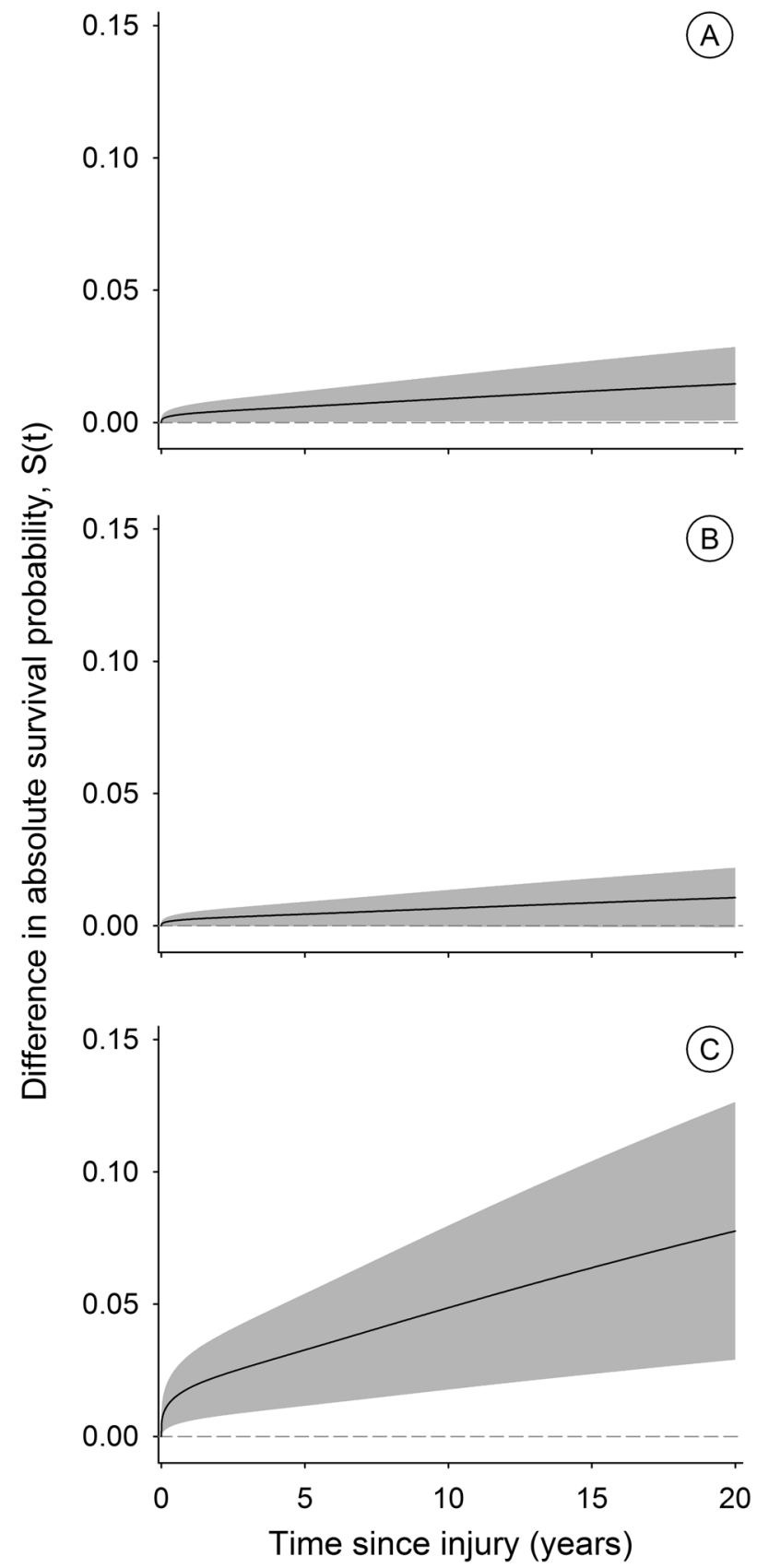

Fig. 2 Differences in absolute survival probabilities according to lesion level and completeness. All figures include incomplete paraplegia as the group of reference. The gray area on either side of the line represents the $95 \%$ confidence interval. a compares the reference group with complete paraplegia; b compares the reference group with incomplete tetraplegia; c compares the reference group with complete tetraplegia. Interpretation: The absolute difference can be interpreted as a percent, for example, 0.04 equates to a $4 \%$ difference. The positive increase of the absolute difference, for example in (c), is indicative of an absolute survival advantage for incomplete paraplegic lesions as compared to complete tetraplegic lesions. The continued upwards trend implies a lack of stabilization (i.e., a continued augmentation in the absolute difference between survival probabilities) acute mortality as 30 days post-injury, and commences time-at-risk with admission to first rehabilitation [3]. Other studies stratify time-at-risk by two-years and more than two-years post-injury [23] or one year post injury [24].

Approaches to statistical analyses in SCI literature vary considerably, as evidenced in a recent systematic review and meta-analysis [4], with some studies reporting HRs, others odds ratios, or relative risks. Moving forward, a standard approach should be adopted that uses advanced, up-to-date statistical techniques, which adequately take into account time-dependent exposures and long-term follow-up to ensure accurate estimates of survival and mortality outcomes, as well as cohesion across SCI literature for ensuring comparable estimates. The flexible parametric survival model methodology employed within this paper is a modern and highly relevant approach often preferable to Cox regression due to the associated issues, for example, regarding predictions of life expectancy, and nonproportional hazards [11]. Additionally, implementation of an individual-based meta-analysis - using data from multiple studies and sources-would ensure standardized stratification, inclusion and exclusion criteria, and mortality definitions [25].

\section{Strengths \& limitations}

This study reports results from a longitudinal cohort study with a clearly defined source population, and includes more than 2400 included cases of TSCI and 376 deaths. However, given that information on AIS scores was only available post-2000, there were a limited number of cases and deaths to include in the analyses. The reduced number of cases, and thereby resulting limited power, may have contributed to the higher risk of mortality estimated for persons with a C5-C8 AIS A, B, or C lesion compared to those with a C1-C4 AIS A, B, or C lesion. This is contrary to estimates from many-albeit not all $[2,26]$ — previous studies [4]. However, given the wide confidence intervals, no concrete conclusions regarding this pattern can be made for this study. Another issue related to data availability in the present study, is the potential bias related to loss to follow-up $(N=171 ; 7.1 \%)$ (e.g., selection bias due to younger individuals being less likely to stay in contact with the rehabilitation clinic and thereby having a higher likelihood of becoming LTFU due to undocumented address changes that would have impacted tracing methodology). To overcome this potential issue, sensitivity analyses including inverse probability weights were employed (Supplementary table). No significant differences in estimates were observed. However, use of IPWs does not account for informative censoring where, for example, persons LTFU may have a differential risk of mortality not related to collected covariates. To correctly adjust estimates, 
a pattern-mixture approach, for instance, could be used to inform mortality risk of those censored [27].

In a previous study assessing the coverage of the SwiSCI Medical Records study with respect to TSCI, it was shown that SwiSCI likely underrepresents less severe cases, as well as the elderly population; this potentially affects the generalizability of the study, and subsequent external validity $[28,29]$. In particular, caution should be taken when interpreting overall estimates of survival as they could be under- or overestimated depending on the added risk from underrepresented groups. However, this study covers the population receiving specialized rehabilitation, as SwiSCI includes all recognized centers. Presented estimates can thus be said to be internally valid and generalizable to the Swiss TSCI population admitted to specialized first rehabilitation. Another limitation of the present study is the absence of information on known contributing risk factors for mortality. Identification of major mediating factors down-stream from SCI-specific characteristics (e.g., completeness of lesion) is important for identifying critical, modifiable factors contributing to potentially preventable mortality [30]; for example, secondary HCs are known to contribute to risk of mortality [19, 31]. In the next steps, cause of death information is needed for cause-specific mortality analyses, as to further evaluate the role of secondary HCs in risk of mortality.

\section{Conclusion}

This study provides evidence of disparities in mortality and survival outcomes between SCI-specific characteristics. Estimates of mortality and survival provided within this study provide an initial evidence base for informing resource allocation and future research directions, whereas future research is needed that focuses on the identification of modifiable contextual factors contributing to risk differentials between SCI characteristics. Analysis of causespecific mortality is the next step needed towards identifying modifiable risk factors contributing to this disparity. Additionally, in medical record studies-or other studies based on routinely collected data, for which information of secondary $\mathrm{HCs}$ is not available-analysis of cause-specific mortality may offer insight regarding the influential secondary HCs contributing to the aforementioned disparities within the SCI population.

Authors' contributions JDC and MWGB were responsible for conceptual framing of the present study. MWGB further provided statistical support as well as critical feedback on manuscript content. HPG, $\mathrm{KH}, \mathrm{XJ}$, and SM provided clinical support and feedback of the present manuscript, as well as support in data collection at their respective clinics. AM provided statistical support for analyses, as well as critical evaluation of statistical methods implemented. Finally, JDC was responsible for all analyses, drafting, and finalization of the present manuscript.

\section{Compliance with ethical standards}

Conflict of interest The authors declare that they have no conflict of interest.

\section{References}

1. World Health Organization. International perspectives on spinal cord injury. Malta: WHO; 2013.

2. Middleton JW, Dayton A, Walsh J, Rutkowski SB, Leong G, Duong S. Life expectancy after spinal cord injury: a 50-year study. Spinal Cord. 2012;50:803-11.

3. Hagen EM, Lie SA, Rekand T, Gilhus NE, Gronning M. Mortality after traumatic spinal cord injury: 50 years of follow-up. J Neurol Neurosurg Psychiatry. 2010;81:368-73.

4. Chamberlain JD, Meier S, Mader L, von Groote PM, Brinkhof MW. Mortality and longevity after a spinal cord injury: systematic review and meta-analysis. Neuroepidemiology. 2015;44:182-98.

5. Brinkhof MW, Al-Khodairy A, Eriks-Hoogland I, Fekete C, Hinrichs $\mathrm{T}$, Hund-Georgiadis $\mathrm{M}$, et al. Health conditions in people with spinal cord injury: Contemporary evidence from a population-based community survey in Switzerland. J Rehabil Med. 2016;48:197-209.

6. Post MW, Brinkhof MW, von Elm E, Boldt C, Brach M, Fekete C, et al. Design of the Swiss spinal cord injury cohort study. Am J Phys Med Rehabil. 2011;90:S5-16.

7. Chamberlain JD, Deriaz O, Hund-Georgiadis M, Meier S, ScheelSailer A, Schubert M, et al. Epidemiology and contemporary risk profile of traumatic spinal cord injury in Switzerland. Inj Epidemiol. 2015;2:28.

8. Brinkhof MW, Fekete C, Chamberlain JD, Post MW, Gemperli A. Swiss national community survey on functioning after spinal cord injury: protocol, characteristics of participants and determinants of non-response. J Rehabil Med. 2016;48:120-30.

9. DeVivo MJ, Biering-Sorensen F, New P, Chen Y. Standardization of data analysis and reporting of results from the International Spinal Cord Injury Core Data Set. Spinal Cord. 2011;49:596-9.

10. Kuehni CE, Rueegg CS, Michel G, Rebholz CE, Strippoli MP, Niggli FK, et al. Cohort profile: the Swiss childhood cancer survivor study. Int J Epidemiol. 2012;41:1553-64.

11. Royston P, Lambert PC: Flexible parametric survival analysis using Stata: beyond the Cox model. Texas: Stata Press; 2011.

12. StataCorp. Stata statistical software: release 15. StataCorp LP: Texas; 2015.

13. Ahoniemi E, Pohjolainen T, Kautiainen H. Survival after spinal cord injury in Finland. J Rehabil Med. 2011;43:481-5.

14. Pavlicek D, Krebs J, Capossela S, Bertolo A, Engelhardt B, Pannek J, et al. Immunosenescence in persons with spinal cord injury in relation to urinary tract infections-a cross-sectional study. Immun Ageing. 2017;14:22.

15. Riegger T, Conrad S, Schluesener HJ, Kaps HP, Badke A, Baron $\mathrm{C}$, et al. Immune depression syndrome following human spinal cord injury (SCI): a pilot study. Neuroscience. 2009; 158:1194-9.

16. Brommer B, Engel O, Kopp MA, Watzlawick R, Muller S, Pruss H, et al. Spinal cord injury-induced immune deficiency syndrome enhances infection susceptibility dependent on lesion level. Brain. 2016;139:692-707.

17. Jensen MP, Truitt AR, Schomer KG, Yorkston KM, Baylor C, Molton IR. Frequency and age effects of secondary health 
conditions in individuals with spinal cord injury: a scoping review. Spinal Cord. 2013;51:882-92.

18. Krause JS, Cao Y, DeVivo MJ, DiPiro ND. Risk and protective factors for cause-specific mortality after spinal cord injury. Arch Phys Med Rehabil. 2016;97:1669-78.

19. Krause JS, Saunders LL. Health, secondary conditions, and life expectancy after spinal cord injury. Arch Phys Med Rehabil. 2011;92:1770-5.

20. Redelings MD, Lee NE, Sorvillo F. Pressure ulcers: more lethal than we thought? Adv Skin Wound Care. 2005;18:367-72.

21. Edsberg LE, Black JM, Goldberg M, McNichol L, Moore L, Sieggreen M. Revised national pressure ulcer advisory panel pressure injury staging system: revised pressure injury staging system. J Wound Ostomy Cont Nurs. 2016;43:585-97.

22. Cao Y, Selassie AW, Krause JS. Risk of death after hospital discharge with traumatic spinal cord injury: a population-based analysis, 1998-2009. Arch Phys Med Rehabil. 2013;94:1054-61.

23. Sabre L, Remmer S, Adams A, Vali M, Rekand T, Asser T, et al. Impact of fatal cases on the epidemiology of traumatic spinal cord injury in Estonia. Eur J Neurol. 2015;22:768-72.

24. Savic G, DeVivo MJ, Frankel HL, Jamous MA, Soni BM, Charlifue S. Long-term survival after traumatic spinal cord injury: a 70-year British study. Spinal Cord. 2017;55:651-8.
25. Stewart LA, Tierney JF. To IPD or not to IPD? Advantages and disadvantages of systematic reviews using individual patient data. Eval Health Prof. 2002;25:76-97.

26. Cao Y, Krause JS, Dipiro N. Risk factors for mortality after spinal cord injury in the USA. Spinal Cord. 2013;51:413-8.

27. Brinkhof MW, Spycher BD, Yiannoutsos C, Weigel R, Wood R, Messou E, et al. Adjusting mortality for loss to follow-up: analysis of five ART programmes in sub-Saharan Africa. PLoS ONE. 2010;5:e14149.

28. Chamberlain JD, Ronca E, Brinkhof MW. Estimating the incidence of traumatic spinal cord injuries in Switzerland: using administrative data to identify potential coverage error in a cohort study. Swiss Med Wkly. 2017;147:w14430.

29. Kukull WA, Ganguli M. Generalizability: the trees, the forest, and the low-hanging fruit. Neurology. 2012;78:1886-91.

30. Corraini P, Olsen M, Pedersen L, Dekkers OM, Vandenbroucke JP. Effect modification, interaction and mediation: an overview of theoretical insights for clinical investigators. Clin Epidemiol. 2017;9:331-8.

31. Krause JS, Saunders LL. Risk of mortality and life expectancy after spinal cord injury: the role of health behaviors and participation. Top Spinal Cord Inj Rehabil. 2010;16:53-60. 